\title{
21-cm line observations of galaxies from Kazarian's lists ${ }^{\star}$
}

\author{
V.S. Tamazian ${ }^{1}$, G. Theureau ${ }^{2}$, and N. Coudreau-Durand ${ }^{2}$ \\ 1 Astronomical Observatory "Ramon Maria Aller", University of Santiago de Compostela, P.O. Box 197, 15706 Santiago de \\ Compostela, Spain \\ ${ }^{2}$ Observatoire de Paris-Meudon, ARPEGES/CNRS URA1757, F-92195 Meudon Principal Cedex, France
}

Received January 24; accepted April 15, 1997

\begin{abstract}
The 21-cm neutral hydrogen line has been measured for the first time in 39 non-Seyfert type galaxies from Kazarian list, with the Nançay radiotelescope. The line profiles, widths at $20 \%$ and $50 \%$ of the peak intensity, radial velocities as well as total fluxes are presented. The values of radial velocity are in fairly good agreement with those obtained from optical spectra. The width values at $20 \%$ of the peak vary in wide range from $113 \mathrm{~km} \mathrm{~s}^{-1}$ (KAZ 579) to $608 \mathrm{~km} \mathrm{~s}^{-1}$ for KAZ 566. Nine of our objects remained undetected, whether their HI-flux was too faint, the integration time too short, or the frequency sighted wrong. However, for part of them, there were positive hint of detection. Comments on individual objects are given.
\end{abstract}

Key words: galaxies: general — radio lines: galaxies

\section{Introduction}

The list of new galaxies with UV-excess published by Kazarian (1979a) and Kazarian \& Kazarian (1982) contains 580 objects exhibiting large variety of morphological (Kazarian et al. 1981; Egiazarian 1983; Tamazian 1987) and spectral (Kazarian 1979a,b; Kazarian et al. 1981; Kazarian 1987; Kazarian \& Kazarian 1987; Kazarian \& Tamazian 1993) properties.

More than $80 \%$ of galaxies show strong emission lines of Balmer series and well known forbidden lines of $[\mathrm{OII}],[\mathrm{OIII}],[\mathrm{NII}],[\mathrm{SII}]$ etc. This is usualy observed in Markarian galaxies (Weedman \& Khachikian 1969; Arakelian et al. 1972) as well as in other galaxies showing unusual excess of UV radiation - blue Haro galaxies (du Puy 1970), ultraviolet-excess galaxies from Kiso survey

Send offprint requests to: G. Theureau

* Tables 1 and 3 are only available in electronic form at CDS. Table 2 is also available in electronic form at CDS via anonymous ftp to cdsarc.u-strasbg.fr (130.79.128.5) or via http://cdsweb.u-strasbg.fr/Abstract.html
(Takase \& Miyauchi-Isobe 1984). This implies significant gas amounts excited by unusually high relative number of hot $\mathrm{O}, \mathrm{B}$ stars in vast majority of objects included in above mentioned lists.

$U B V$-photometric study of some galaxies from Kazarian list (Tamazian 1983; Tamazian 1984; Kazarian \& Tamazian 1984) shows from their colors distribution that they are similar to non-Seyfert Markarian galaxies (Huchra 1977) and tend to be bluer when approaching their central parts. Some galaxies from Kazarian lists have been observed occasionally in different radio wavelengths with different instruments but no systematic study of these galaxies has been done before.

Only very few number of galaxies has been observed at 21-cm: for example, KAZ 546 (Paturel, unpublished), neutral hydrogen study of Haro galaxies (Bottinelli et al. 1973a), Markarian (Bottinelli et al. 1973b, 1975) and Kiso UV-excess galaxies (Maehara et al. 1988), also using the Nançay radiotelescope. These studies allow to obtain valuable information on astrophysical parameters of these galaxies: systemic radial velocity, amplitude of internal rotation, HI gas content.

There are various families of galaxies among Kazarian lists (elliptical and lenticular galaxies with emission lines and strong UV-excess; blue dwarfs, possibly metal deficient; giant starburst galaxies with high infrared luminosity) which can be selected as best candidates to have measurable HI flux. Thus, HI observations of these galaxies is interesting both to determine important astrophysical parameters and to compare their properties with those of galaxies from other lists.

For technical reasons we selected among Kazarian's lists those galaxies having radial velocities smaller than $12000 \mathrm{~km} \mathrm{~s}^{-1}$ (known from different sources listed in Table 1).

\section{Observations}

The observations have been performed with the transitmeridian Nançay (France) radiotelescope, which has a collecting area of $6912 \mathrm{~m}^{2}(200 \times 34.56)$. 
The half-power beam width at $21-\mathrm{cm}$ is 3.6 arcmin $(\mathrm{EW}) \times 22 \operatorname{arcmin}(\mathrm{NS})$ (at zero declination). The minimal system temperature at $\delta=15^{\circ}$ is about $37 \mathrm{~K}$ in both horizontal and vertical polarizations. The gain of the antenna has been calibrated according to Fouqué et al. (1990).

The spectrometer is a 1024-channels autocorrelator of 6.4 MHz total bandwidth. The spacing of the channels corresponds to $2.6 \mathrm{~km} \mathrm{~s}^{-1}$ at $21 \mathrm{~cm}$ with a bank of 512 channels in each polarization (horizontal and vertical). After boxcar smoothing the final resolution is typically $12 \mathrm{~km} \mathrm{~s}^{-1}$. Each final spectrum corresponds to the integration on $n$ cycles of 4 minutes each (on/off beam switch mode, the size of the beam, and the position of the comparison field varying with declination). After rejection of disturbed cycles (interferences, noisy baseline), the spectrum is straightened by fitting a polynomial function to the external parts of the line (1st to 6th degree), and the maximum intensity of the line is estimated.

Observed radial velocities correspond to the median point of the 21-cm line profile measured at $20 \%$ level of maximum intensity. The internal mean error $\sigma\left(V_{20}\right)$ on $V_{20}$ is calculated according to Fouqué et al. (1990) from the actual spectral resolution $r$, the slope $a=\left(W_{20}+W_{50}\right) / 2$ of the line profile, and the signal to noise ratio $S / N$ :

$\sigma\left(V_{20}\right)=\frac{4 \cdot r^{\frac{1}{2}} \cdot a^{\frac{1}{2}}}{S / N}$

Line widths are measured on the observed profile at two standard levels corresponding to $20 \%$ and $50 \%$ of the maximum intensity of the line. These widths $W_{20}$ and $W_{50}$ are first corrected for spectral resolution effect in agreement with Bottinelli et al. (1990), and further corrected for internal velocity dispersion assuming an isotropic distribution of the non-circular motions and a nearly gaussian velocity distribution (Fouqué et al. 1990):

$W_{\text {c }}=W^{2}+w t^{2}\left(1-2 \mathrm{e}^{-W^{2} / w^{2}}\right)-2 W \cdot w t\left(1-\mathrm{e}^{-W^{2} / w^{2}}\right)$

where $w t=2 \sigma_{z} \cdot k(l)\left(\sigma_{z}=12 \mathrm{~km} \mathrm{~s}^{-1}, k(20)=1.96\right.$ and $k(50)=1.13)$, and $w$ is equal to 120 and $100 \mathrm{~km} \mathrm{~s}^{-1}$ for the levels $20 \%$ and $50 \%$ respectively.

The mean errors are respectively $3 \sigma\left(V_{20}\right)$ and $2 \sigma\left(V_{20}\right)$ for the $20 \%$ and $50 \%$ widths, according to Fouqué et al. (1990).

Integrated $\mathrm{HI}$ fluxes are expressed in $\mathrm{Jy} \mathrm{km} \mathrm{s}^{-1}$ and corrected for beam-filling, when both photometric diameter and axis ratios were known, using the formula given by Bottinelli et al. (1990):

$F_{\mathrm{HI}}=R \cdot F$

where

$R=\left[1+\frac{D_{25}^{2} \sin ^{2} \beta+d_{25}^{2} \cos ^{2} \beta}{22}\right]^{\frac{1}{2}}$ and $\beta$ is the position angle of the galaxy defined northeastwards, $D_{25}$ and $d_{25}$ are the photometric major and minor axis respectively.

These fluxes have been calibrated by using a set of 6 close calibrator galaxies, whose HI-fluxes were accurately measured by Fouqué (1982) and which have been regularly observed during all the period covered by our survey (june 96 - November 96). This calibration is done by monitoring the performance of the Nançay system with time, in both horizontal and vertical polarization separately, in order to get for each observation the better HI flux measurement (see Theureau et al. 1997 for more details).

Main optical parameters of observed galaxies are given in Table 1. When more than one measurement of optical radial velocity is available from literature we list its value extracted from the Lyon-Meudon Extragalactic Database (LEDA, Paturel et al. 1997) whereas in last column all references on radial velocity measurements for each galaxy are given.

Note that all astrophysical data extracted from LEDA are reduced to a common system according to well defined reduction formulae leading to mean homogenized parameters. Apparent diameters are reduced to the standard system of the isophote at the brightness of $25 B$-mag $\operatorname{arcsec}^{-2}$. Apparent $B$-magnitudes are reduced to the RC3 system. Both are corrected for extinction, inclination (i.e. opacity), and redshift effect. For extinction correction on $B$-magnitude, or $\log D_{25}$ diameters, the RC2 system has been used. Opacity corrections in $B$-band are calculated according to Bottinelli et al. (1995).

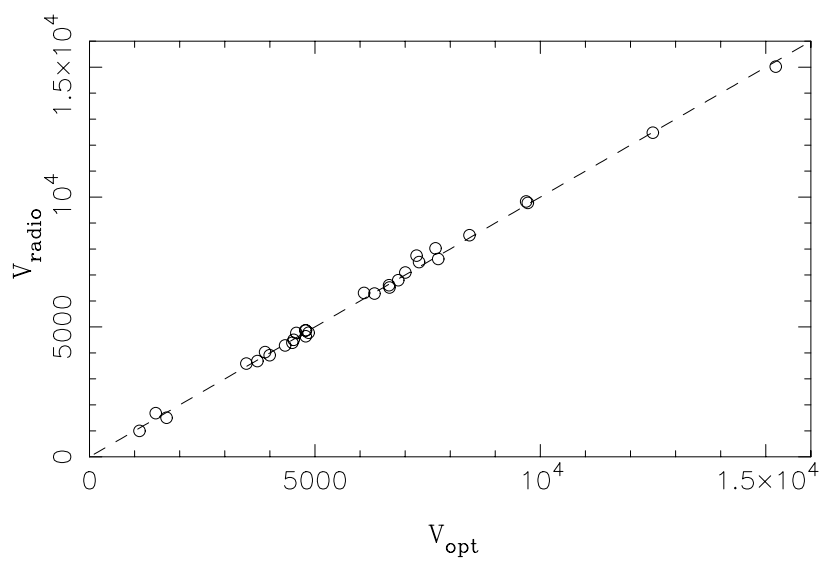

Fig. 1. Comparison of optical heliocentric radial velocities (values from LEDA, see Table 1) with our HI velocity measurements with the Nançay radiotelescope (in $\mathrm{km} \mathrm{s}^{-1}$ ): the dashed line corresponds to $V_{21}=V_{\mathrm{opt}}$

The observed 21-cm line profiles are given in Fig. 2. All derived HI parameters, corrected to the optical velocity scale, are listed in Table 2 . Our 21-cm radial velocities appear in very good agreement with optical ones as shown in Fig. 1, where the line corresponding to $V_{21}=V_{\mathrm{opt}}$ is 
drawn. The values of the rms noise, averaged outside the line profile indicate a mean $\mathrm{rms}$ noise of about $2 \mathrm{mJy}$ at resolution $12 \mathrm{~km} \mathrm{~s}^{-1}$. Discussions of parameters and their detailed comparison with those from other similar lists of galaxies will be presented in a forthcoming paper.

\section{Individual comments on galaxies}

KAZ 001: Emission lines of [SII], [OIII],[OII], $\mathrm{H}_{\alpha}$ and $\mathrm{H}_{\beta}$. The Balmer lines from $\mathrm{H}_{\gamma}$ to $\mathrm{H}_{15}$ as well as $\mathrm{Ca}$ II $\lambda 3934$ in absorption. More details on optical spectra are given by Kazarian \& Khachikian (1974), and Kazarian et al. (1982). It should be noted that the values of the maximum rotational velocity (interpreted as the mass within a certain radius) estimated from both optical and radio measurements can not be compared because they do not refer to the same scale within the disc (only the central parts, within $2 \mathrm{kpc}$, contribute to the optical measurement, as the globality of the HI disc enters the HI profile width). The $21-\mathrm{cm}$ line profile is very well defined. KAZ 003: Starburst galaxy with spectrum very similar to that of KAZ 1 (Kazarian \& Khachikian 1974). More details in Kazarian et al. (1982). The 21-cm line profile is very well defined with a double-peaked shape typical of spirals.

KAZ 004: Starburst galaxy with emission lines of [SII] $\lambda \lambda 6731 / 17$ and [NII] $\lambda \lambda 6584 / 48, \mathrm{H}_{\alpha},[\mathrm{OIII}] \lambda \lambda$ $5007 / 4959, \mathrm{H}_{\beta}$ and $\mathrm{H}_{\gamma}$ observed in the spectra. The lines of Balmer series from $\mathrm{H}_{\delta}$ to $\mathrm{H}_{15}$ are observed in absorption. This galaxy has been observed 121 cycles but only 73 of them resulted usable due to many interferences quite usual in this low frequency range. Too noisy spectrum.

KAZ 006: Emission lines [SII] $\lambda \lambda$ 6731/17, [NII] $\lambda \lambda$ $6584 / 48$ and $\mathrm{H}_{\alpha}$ are observed in the spectrum. The redshift given by Kazarian (1979a) is $z=0.0267$ ( $V$ (opt) $=$ $8010 \mathrm{~km} / \mathrm{s}$ ) and differs from that presented in Table 1. Only 15 cycles were available, there is a hint of HI detection near $V=6000 \mathrm{~km} / \mathrm{s}$ but the signal is too faint compared with the noise.

KAZ 016: On the plates taken in prime focus of $2.6 \mathrm{~m}$ telescope of Byurakan Observatory (scale $21^{\prime \prime} / \mathrm{mm}$ ) two circular condensations separated by $8^{\prime \prime}$ are seen. Morphological type given in LEDA is Sc, but it is classified as irregular by Kazarian (1979a). Pair galaxy: HI confusion with companion UGC 41, which is a Sc galaxy with same magnitude, same inclination, and approximately same velocity $(V($ opt $)=6597 \mathrm{~km} / \mathrm{s},[18])$. Steep-sided HI profile typical of a spiral galaxy, but the two profiles are most probably superimposed (this could explain the peculiar shape of the high velocity peak).

KAZ 018: According to Kazarian this is an irregular galaxy with bright central region measuring $3^{\prime \prime} \times 4^{\prime \prime}$ which could be taken for a bar (morphological type in LEDA is SBbc). $U B V$-photometry carried out by Tamazian (1983) shows that the nucleus is rather blue $(U-B=-0.11$,
$B-V=+0.43)$. Integral color $U-B=0.23, B-V=$ +0.69 . Assymetrical HI profile.

KAZ 019: Spiral with bright nucleus of about $4^{\prime \prime}$. According to Tamazian (1983), the nucleus is very blue $(U-B=-0.31, B-V=+0.36)$, integral color of galaxy $U-B=-0.02, B-V=+0.49$. Well defined doublepeaked profile.

KAZ 023: The morphological type given in original list is elliptical whereas in LEDA it is described as Sc. Integral color $U-B=+0.25, B-V=+0.68$ but the nucleus is much bluer $-U-B=-0.14, B-V=+0.48$ (Tamazian 1983). Well defined double-peaked HI profile.

KAZ 024: Described in original list as elliptical with compact nucleus. As in case of KAZ 23 we adopted a Sc morphological type according to LEDA. HI measured radial velocity confirms the value obtained by Focardi et al. (1982) $-6321 \mathrm{~km} / \mathrm{s}$ and differs from that obtained by Huchra et al. (1983) given in Table 1 .

KAZ 026: Very strong but narrow emission lines in spectra, which are investigated in details by Shuder \& Osterbrock (1981) and Kazarian \& Tamazian (1982a). This object is very interesting and well investigated (see references in Table 1). Strong IRAS source (IRAS 07204+3332), FIR magnitude is 11.2 (IRAS Point Source Catalogue, 1988). Extremely blue with integral $U-B=$ $-0.86, B-V=+0.60$, but certainly is not Seyfert type galaxy. HI profile is asymmetrical, due to an interference appearing only on part of the cycles (peak at $V=4100 \mathrm{~km} / \mathrm{s}$ ). However, the edges of the profile are not disturbed and the HI flux is only weakly affected.

KAZ 039: Lenticular, with bright nucleus. Our derived radial velocity is $350 \mathrm{~km} / \mathrm{s}$ larger than the optical one obtained by Kazarian (1987). There are four galaxies lying within the Nançay beam: IC 1146 or MCG 12-15-19 (no known velocity, lenticular according to MCG) which might be the weak signal at $V=7150 \mathrm{~km} / \mathrm{s}$ : UGC 10053 and MCG 12-15-21 with known (optical) radial velocities $11200 \mathrm{~km} / \mathrm{s}$ and $10103 \mathrm{~km} / \mathrm{s}$ respectively (LEDA) which cannot affect the detected signal: and MCG 12-1518 which is elliptical $(T=-5)$ with unkown velocity but probably with a very poor HI content.

KAZ 047: Compact dwarf galaxy surrounded by an heterogeneous extended halo. Very faint HI signal around the optical velocity $V=4640 \mathrm{~km} / \mathrm{s}$.

KAZ 052: Early spiral with bright nucleus. HI confusion due to NGC 6068 (Sbc galaxy, according to de Vaucouleurs et al. 1991), which was already measured at 21-cm with $V=3975 \mathrm{~km} / \mathrm{s}$ (Davis \& Seaquist 1983; see also [2][40][26] for optical measurements). Poor S/N ratio and very uncertain edges of the line profile.

KAZ 060: Early spiral, 21-cm integration time of 80 cycles, but below detection level $(S / N=1.5$, for $\sigma \simeq$ $2.7 \mathrm{mJy}$ ). However, a hint of detection appears between $V=8400$ and $9100 \mathrm{~km} / \mathrm{s}$ around the optical radial velocity listed in Table 1. 
KAZ 063: Irregular, bright starlike condensation observed on the southern edge. Pair with UGC 10324 (type Sd) with $V$ (opt.) $=7016 \mathrm{~km} / \mathrm{s}([43])$ also detected within the beam as a separate signal. The HI profile of KAZ 063 is well distinguishable at lower velocities around $V=$ $6500 \mathrm{~km} / \mathrm{s}$.

KAZ 065: Spiral with bright nucleus $\left(4^{\prime \prime}\right)$. One of the arms ends to south with starlike condensations. Triangular HI profile. IC 1216 (no known velocity, Sc galaxy according to de Vaucouleurs et al. 1991) is also within the Nançay beam but no distorsion is observed. Our HI velocity confirms the value $7736 \mathrm{~km} / \mathrm{s}$ obtained by Strauss \& Huchra (1988), but differs from $V=7255 \mathrm{~km} / \mathrm{s}$ given by Kazarian (1987).

KAZ 069: Lenticular; a dark lane of width $1^{\prime \prime}$ passing through the bright central part perpendicular to the lens. Wide HI line detected with $S / N=3$. Very close object with a small HI content.

KAZ 098: Elliptical with extension attached to the south-west edge, classified as a very peculiar object in UGC. HI signal well detected.

KAZ 120: Spiral galaxy with starlike condensation on the edge of the northern arm. Very poor HI detection $(S / N=1.7$, for $\sigma \simeq 7.4 \mathrm{mJy})$, however, the shape of the HI line is identified: a hint of signal between 4600 and $5100 \mathrm{~km} / \mathrm{s}$ around the optical radial velocity $(4800 \mathrm{~km} / \mathrm{s})$ listed in Table 1.

KAZ 198: Described as irregular, consisting of separate regions based on direct images taken on $2.6 \mathrm{~m}$ telescope of Byurakan observatory, whereas in LEDA appears as Sc. Emission lines of [SII], [OIII], $\mathrm{H}_{\alpha}$ and $\mathrm{H}_{\beta}$ are present in the spectra. Total gas mass is about $6.410^{9}$ solar mass estimated by Kazarian (1984). Unusual HI profile with several separate peaks. HI confusion with the faceon Sa galaxy MCG 11-22-47 known to have $V$ (opt.) = $4108+/-100 \mathrm{~km} / \mathrm{s}([23][25][26][48])$, then, a corresponding narrow HI linewidth with a relatively high $S / N$ ratio is expected. It could be the intermediate peak (near $V=4300 \mathrm{~km} / \mathrm{s}$ ) seen in the global observed wide profile. If, according to LEDA, KAZ 198 is an edge-on spiral, it is expected to have a wide $\mathrm{HI}$ profile, and a rather important HI flux: it probably contributes to the observed total width.

KAZ 201: One of the most faintest galaxies $\left(B_{\mathrm{t}}=18.0\right)$. Lenticular. KAZ $202\left(B_{\mathrm{t}}=17.0\right)$ in the off-field of comparison, but does not seem to disturb the signal. However, the HI profile is noisy, the low velocity edge is badly defined.

KAZ 211: Lenticular galaxy. Clear HI detection with an ill-defined edge at high velocity.

KAZ 216: Irregular, has six starlike knots.

KAZ 228: According to original list, it is a spiral galaxy with a bright circular nucleus (2.6 $\mathrm{m}$ telescope), but in LEDA it appears as elliptical. Faint HI signal $(\approx 2 \mathrm{mJy})$ detected at $2.9 \sigma$ thanks to very long integration time (212 cycles). Although NGC 7323 is within the beam, no
HI confusion is possible because of very different radial velocity $V=5600 \mathrm{~km} / \mathrm{s}([10][17][19][20][26])$.

KAZ 236: Emission lines $[\mathrm{NII}] \lambda \lambda 6584 / 48, \mathrm{H}_{\alpha},[\mathrm{OIII}] \lambda \lambda$ $5007 / 4959$ and $\mathrm{H}_{\beta}$ are observed. Detailed spectral study (Kazarian \& Tamazian 1982b) showed the lack of dust in this galaxy. We observed it in HI at $V=8080 \mathrm{~km} / \mathrm{s}$ as listed in Palumbo et al. (1983) (see Table 1). In fact, there is a single determination of its radial velocity which is $V=6060 \mathrm{~km} / \mathrm{s}(z=0.020)$, as given by Kazarian (1979) or Kazarian \& Tamazian (1982b). The 21-cm spectrum shown in Fig. 2 is consequently not centered on the right velocity.

KAZ 248: Faint HI signal, suspected between 8900 and $9600 \mathrm{~km} / \mathrm{s}$ around the optical radial velocity, but below detection level $(2 \sigma \simeq 5.2 \mathrm{mJy})$

KAZ 282: Faint but well determined HI signal.

KAZ 291: Elliptical. No significant HI signal detected above $2 \sigma \simeq 2.8$ mJy. KAZ 292 at the edge of the Nançay beam.

KAZ 305: Compact galaxy surrounded by faint halo. No HI signal detected above the level $2 \sigma \simeq 3.6 \mathrm{mJy}$.

KAZ 347: According to Humason et al. (1956) NGC 7770 has a strong ultraviolet continuum of early type, broad and weak absorption lines of the Balmer series as well as weak emission at $\lambda 3727 \mathrm{~A}[\mathrm{O} \mathrm{II}]$ which is possibly inclined. In pair with NGC 7771 located at 1.1 arcmin and disturbed; member of NGC 7769 group. Investigated photometricaly by Tamazian (1984). Integral colors $U-B=$ $+0.13, B-V=+0.49$, but the bright nucleus is bluer with $U-B=-0.27, B-V=+0.34$. According to Humason et al. (1956) the radial velocity is $V=4338 \mathrm{~km} / \mathrm{s}$, but more precise measurements indicate smaller radial velocity $V=4077 \mathrm{~km} / \mathrm{s}$ (see Table 1). Our HI line appears disturbed. NGC 7771 ( $V($ opt $)=4311 \mathrm{~km} / \mathrm{s}$, [7] [10] [17] [19] [22] [26] [29] [30] [37] [38] [42] [45]) is located within the Nançay beam and there is HI confusion: what is expected is a narrow emission from the face-on galaxy KAZ $347=$ NGC 7770 (around $V \simeq 4300 \mathrm{~km} / \mathrm{s}$ ) superimposed on a relatively wide HI emission, centered at a nearby velocity, from the large inclined EW spiral NGC 7771. Note that Schneider et al. (1986) have observed the system NGC $7769 / 7770 / 7771$ at Arecibo (HPSW = 3.6 arcmin) and state that NGC 7770 "has a broad peculiar wing on the high-velocity side of its HI profile". This feature is also present here and is typical of interacting galaxies.

KAZ 368: Lenticular, with bright central region. No significant $\mathrm{HI}$ signal at the level $2 \sigma \simeq 3.4 \mathrm{mJy}$. Some hint of detection between 6000 and $6500 \mathrm{~km} / \mathrm{s}$ around the optical radial velocity.

KAZ 445: Lenticular, probably with starlike nucleus. Well defined double-peaked HI profile, already published by Bottinelli et al. (1993).

KAZ 512: Starlike with very strong UV excess. Bright FIR source of magnitude 12. Despite very far distance, hint of $\mathrm{HI}$ detection around $15000 \mathrm{~km} / \mathrm{s}$ near the optical radial velocity with $S / N=2.3$. 
KAZ 520: Described as elliptical galaxy in original list, but Sc from LEDA. No significant HI signal at the level $2 \sigma \simeq 2.8 \mathrm{mJy}$.

KAZ 524: Spiral; another fainter elliptic galaxy at $50^{\prime \prime}$ to the south. HI signal confused with that from KCPG $557 \mathrm{~A}(V=8407 \mathrm{~km} / \mathrm{s}$, Tifft 1982). The two HI profiles are superimposed: probably interacting galaxies.

KAZ 544: HI signal is very well detected. Although ESO $533-48(V=5600 \mathrm{~km} / \mathrm{s},[8][35][49])$ was at the edge of the Nançay beam, no HI confusion.

KAZ 546: Previously observed at 21-cm with $W(20)=$ $283 \mathrm{~km} / \mathrm{s}$ and $W(50)=271 \mathrm{~km} / \mathrm{s}$ at $V=7314 \mathrm{~km} / \mathrm{s}(\mathrm{G}$. Paturel, unpublished). Our result is significantly different for the radial velocity $(\Delta V=180 \mathrm{~km} / \mathrm{s})$.

KAZ 566: Well defined HI profile for such a far object. KAZ 579: Elliptical galaxy confused with MCG 0-1-14 (very compact galaxy, classified as elliptical by LEDA) at $V$ (opt.) $=7028 \mathrm{~km} / \mathrm{s}([2][24])$. Our derived velocity $V=7099 \mathrm{~km} / \mathrm{s}$ confirms the optical radial velocity $V=7103 \mathrm{~km} / \mathrm{s}$ given in Arakelian et al. (1975).

Acknowledgements. We thank Dr. L. Gouguenheim, and Dr. L. Bottinelli for helpful comments. We have made use of data from Lyon-Meudon Extragalactic Database (LEDA) compiled by the LEDA team at the CRAL-Observatoire de Lyon (France).

\section{References}

Arakelian M.A., Dibays E.A., Esipov V.F., 1972, Astrofizika 8, 334

Arakelian M.A., Dibai E.A., Esipov V.F., 1975a, Astrophys. 11,254

Arakelian M.A., Dibai E.A., Esipov V.F., 1975b, Astrophys. 11,8

Baan W.A., Haschick A.D., Henkel C., 1992, AJ 103, 728

Beers T.C., Forman W., Huchra J.P., Jones C., Gebhardt K., 1991, AJ 102, 1581

Bottinelli L., Chamaraux P., Gouguenheim L., Heidmann J., 1973a, A\&A 29, 217

Bottinelli L., Gouguenheim L., Heidmann J., 1973b , A\&A 22, 281

Bottinelli L., Duflot R., Gouguenheim L., Heidmann J., 1975, A\&A 41, 61

Bottinelli L., Gouguenheim L., Fouqué P., Paturel G., 1990, A\&AS 82, 391

Bottinelli L., et al., 1993, A\&AS 102, 57

Bottinelli L., Gouguenheim L., Paturel G., Teerikorpi P., 1995, A\&A 296, 64

Chengalur J.N., Salpeter E.E., Terzian Y., 1993, ApJ 419, 30

Da Costa L.N., Pellegrini P.S., Davis M., Meiksin A., Sargent W.L.W., Tonry J.L., 1991, ApJS 75, 935

Dahari O., 1985, ApJS 57, 643

Dahari O., 1986, erratum in ApJS 60, 601

Davis L.E., Seaquist E.R., 1983, ApJS 53, 269

Denisyuk E.K., Dostal V.A., 1976, Astronomiceskij Cirkuljar 931, 7

Dennefeld M., Sèvre F., 1984, A\&AS 57, 253

Doroshenko V.T., Terebizh V.Yu., 1975, Astrophys. 11, 422

Egiazarian A., 1983, Astrofizika 19, 631
Fairall A., Jones A., 1991, Southern Redshifts Catalogue, Publications of the Department of Astronomy, University of Cape Town, No. 11 (Optical)

Fisher D., Illingworth G., Franx M., 1995, ApJ 438, 539

Focardi P., Marano B., Vettolani G., 1982, A\&A 113, 15

Fouqué P., 1982, PhD thesis, Paris Observatory

Fouqué P., Bottinelli L., Gouguenheim L., Paturel G., 1990, ApJ 349, 1

Giovanardi C., Salpeter E.E., 1985, ApJS 58, 623

Giovanelli R., Haynes M.P., Myers S.T., Roth J., 1986, AJ 92, 250

Giovanelli R., Haynes M.P., 1993, AJ 105, 1271

Huchra J.P., 1977, ApJS 35, 171

Huchra J., Davis M., Latham D., Tonry J., 1983, ApJS 52, 89

Huchra J.P., Latham D., Da Costa L.N., Pellegrini P.S., Willmer C., 1993, AJ 105, 1637

Humason M.L., Mayall N.U., Sandage A., 1956, AJ 61, 97

IRAS Point Source Catalogue, 1988, Version 2, US Gov. Printing Office, Washington

Karachentsev I.D., Pronik V.I., Chuvaev K.K., 1975, A\&A 41, 375

Karachentsev I.D., Sargent W.L.W., Zimmermann B., 1979, Astrophys. 15, 19

Karachentsev I.D., 1980, ApJS 44, 137

Karachentsev I.D., 1987, Catalogue of pairs of galaxies

Kazarian M.A., 1979a, Astrofizika 15, 5

Kazarian M.A., 1979b, Astrofizika 15, 117

Kazarian M.A., 1984 Astrofizika 20, 24

Kazarian M.A., 1987a, Astrofizika 27, 399

Kazarian M.A., 1987b, Astrofizika 27, 569

Kazarian M.A., Khachikian E.Ye., 1974, Astrofizika 10, 299

Kazarian M.A., Petrosian A.R., Tamazian V.S., 1981, Sov. Astron. Lett. 7, 648

Kazarian M.A., Kazarian E.S., 1982, Astrofizika 18, 512

Kazarian M.A., Khachikian E.Ye., Yegiazarian A.A., 1982 Ap. Space Sci. 82, 105

Kazarian M.A., Kazarian E.S., 1987, Astrofizika 26, 5

Kazarian M.A., Tamazian V.S., 1982a, Astrofizica 18, 192

Kazarian M.A., Tamazian V.S., 1982b, Sov. Astron. Lett. 8, 454

Kazarian M.A., Tamazian V.S., 1984, Sov. Astron. Lett. 7, 815

Kazarian M.A., Tamazian V.S., 1993, Astrofizika 36, 363

Kim D.W., Sanders D.B., Veilleux S., Mazzarella J.M., Soifer B.T., 1995, ApJS 98, 129

Kirshner R.P., Oemler A., Schechter P.L., 1978, AJ 83, 1549

Maehara H., Noguchi T., Takase B., Handa T., 1987, PASJ 39, 393

Maehara H., Hamabe M., Bottinelli L., Gouguenheim L., Heidmann J., Takase B., 1988, PASJ 40, 47

Marano B., Vettolani G., 1982, A\&AS 48, 453

Markarian B.E., Lipovetskii V.A., Stepanian J.A., 1980, Astrophys. 16, 353

Mathewson D.S., Ford V.L., Buchhorn M., 1992, ApJS 81, 413

Maurogordato S., Proust D., Balkowski C., 1991, A\&A 246, 39

Mayall N.U., Vaucouleurs A. de, 1962, AJ 67, 363

Mirabel I.F., Sanders D.B., 1988, ApJ 335, 104

Page T., 1961, Proc Fourth Berkeley Symp on Mathematical Statistics and Probability. University of California Press 3, 277

Page T.L., 1970, ApJ 159, 791 
Table 2.

\begin{tabular}{|c|c|c|c|c|c|c|c|c|c|c|c|c|c|c|}
\hline Kaz.name & $\begin{array}{c}V_{20} \\
\mathrm{~km} \mathrm{~s}^{-1}\end{array}$ & $\mathrm{rms}$ & $\begin{array}{c}W_{20} \\
\mathrm{~km} \mathrm{~s}^{-1}\end{array}$ & rms & $\begin{array}{c}W_{50} \\
\mathrm{~km} \mathrm{~s}^{-1}\end{array}$ & $\mathrm{rms}$ & $\log V_{\mathrm{m}}$ & rms & $\begin{array}{c}F(\mathrm{HI}) \\
\mathrm{Jy} \mathrm{km} \mathrm{s}^{-1}\end{array}$ & \begin{tabular}{|c|}
$F(\mathrm{HI})_{\mathrm{c}}$ \\
$\mathrm{Jy} \mathrm{km} \mathrm{s}^{-1}$
\end{tabular} & rms & $S / N$ & $\begin{array}{c}\sigma \\
\mathrm{mJy}\end{array}$ & Notes \\
\hline KAZ001 & 3589 & 7 & 313 & 20 & 254 & 13 & 2.242 & .003 & 11.7 & 11.9 & 1.2 & 12.1 & 4.6 & \\
\hline KAZ003 & 6308 & 5 & 147 & 16 & 137 & 11 & 2.207 & .117 & 2.9 & 2.9 & .5 & 7.4 & 2.0 & \\
\hline KAZ016 & 6603 & 8 & 262 & 25 & 246 & 16 & 2.049 & .002 & 3.1 & 3.2 & .6 & 5.6 & 2.3 & $\mathrm{c}$ \\
\hline KAZ018 & 4777 & 16 & 372 & 47 & 313 & 31 & 2.242 & .004 & 4.0 & 4.1 & .7 & 5.1 & 2.2 & \\
\hline KAZ019 & 4770 & 4 & 394 & 13 & 377 & 8 & 2.287 & .000 & 9.5 & 9.9 & .8 & 11.2 & 2.1 & \\
\hline KAZ023 & 6796 & 13 & 469 & 39 & 392 & 26 & 2.307 & .002 & 6.2 & 6.6 & 1.1 & 6.9 & 4.3 & \\
\hline KAZ024 & 6286 & 10 & 405 & 30 & 388 & 20 & 2.300 & .001 & 4.2 & 4.3 & .7 & 4.8 & 2.0 & \\
\hline KAZ026 & 4031 & 14 & 282 & 43 & 170 & 29 & 2.166 & .012 & 3.1 & 3.1 & .6 & 7.5 & 2.6 & \\
\hline KAZ039 & 8025 & 15 & 224 & 44 & 209 & 29 & & & 1.4 & & .6 & 3.1 & 2.3 & \\
\hline KAZ047 & 4640 & 16 & 578 & 48 & 573 & 32 & & & 1.2 & & .6 & 2.0 & 1.8 & \\
\hline KAZ052 & 3910 & 14 & 170 & 41 & 166 & 27 & & & 1.5 & 1.5 & .9 & 2.2 & 3.7 & \\
\hline KAZ063 & 6519 & 23 & 472 & 70 & 443 & 47 & & & 3.7 & & 1.4 & 2.5 & 4.1 & \\
\hline KAZ065 & 7745 & 10 & 218 & 30 & 185 & 20 & 2.020 & .006 & 6.2 & 6.3 & 1.2 & 6.2 & 4.3 & \\
\hline KAZ069 & 1000 & 23 & 469 & 69 & 427 & 46 & & & 3.4 & 3.5 & .9 & 3.0 & 2.4 & \\
\hline KAZ098 & 4503 & 16 & 377 & 47 & 353 & 31 & & & 3.9 & & 1.1 & 3.4 & 3.2 & \\
\hline KAZ120 & 4866 & 38 & 528 & 115 & 491 & 77 & 2.454 & .010 & 5.8 & 5.9 & 2.8 & 1.7 & 7.5 & \\
\hline KAZ198 & 4393 & 25 & 560 & 76 & 448 & 50 & 2.380 & .004 & 3.5 & 3.7 & 1.0 & 4.3 & 3.6 & $\mathrm{c}$ \\
\hline KAZ201 & 1505 & 8 & 318 & 25 & 314 & 17 & & & 3.1 & & 1.0 & 3.6 & 3.7 & \\
\hline KAZ211 & 1680 & 37 & 566 & 112 & 394 & 74 & & & 6.5 & & 1.6 & 3.6 & 4.4 & \\
\hline KAZ216 & 7620 & 27 & 284 & 80 & 191 & 53 & & & 2.2 & & .7 & 3.7 & 2.6 & \\
\hline KAZ228 & 9777 & 20 & 541 & 59 & 513 & 39 & & & 1.3 & 1.3 & .4 & 2.9 & .9 & \\
\hline KAZ282 & 3685 & 18 & 330 & 53 & 281 & 35 & 2.231 & .007 & 2.8 & 2.8 & .8 & 4.2 & 2.7 & \\
\hline KAZ347 & 4286 & 21 & 536 & 62 & 282 & 41 & & & 6.9 & 7.0 & .7 & 7.8 & 2.0 & $\mathrm{c}$ \\
\hline KAZ445 & 4870 & 25 & 335 & 75 & 235 & 50 & 2.257 & .018 & 4.1 & 4.3 & 1.5 & 4.1 & 6.7 & \\
\hline KAZ512 & 15021 & 10 & 540 & 29 & 540 & 20 & & & 1.2 & & .4 & 2.3 & 1.1 & \\
\hline KAZ524 & 8529 & 18 & 294 & 54 & 242 & 36 & 2.242 & .010 & 1.5 & 1.5 & .4 & 4.2 & 1.2 & c \\
\hline KAZ544 & 9832 & 14 & 307 & 41 & 243 & 27 & 2.274 & .006 & 2.6 & 2.6 & .4 & 6.1 & 1.2 & \\
\hline KAZ546 & 7495 & 5 & 296 & 15 & 281 & 10 & 2.174 & .001 & 5.3 & 5.4 & .6 & 9.3 & 1.8 & \\
\hline KAZ566 & 12479 & 7 & 608 & 21 & 609 & 14 & 2.483 & .000 & 4.4 & 4.5 & 1.0 & 3.2 & 2.2 & \\
\hline KAZ579 & 7099 & 8 & 113 & 23 & 73 & 16 & & & 2.2 & 2.3 & .4 & 8.6 & 2.1 & \\
\hline
\end{tabular}

Column 1: Number from Kazarian lists.

Column 2: Heliocentric systemic radial velocity measured at the mid-point of the $20 \%$ level of peak intensity and its mean error. Column 3: Line width measured at $20 \%$ of the maximum intensity (corrected for resolution and internal motions) with its corresponding mean error.

Column 4: Line width measured at $50 \%$ of the maximum intensity (corrected for resolution and internal motions) with its corresponding mean error.

Column 5: Estimated maximum of rotational velocity calculated according to Paturel et al. (1997) for spiral galaxies with known inclination angle and corresponding mean error.

Column 6: Measured HI flux (in Jy km/s), calibrated according to our set of calibrators (see text).

Column 7: Corrected HI flux (in Jy km/s).

Column 8: Mean error of HI flux.

Column 9: Signal-to-Noise ratio.

Column 10: rms noise outside the line profile (in mJy).

Column 11: HI-confused galaxies are marked with a $c$.

Palumbo G.G.C., Tanzella-Nitti G., Vettolani G., 1983, Catalogue of Radial Velocities of Galaxies. Gordon and Breach

Paturel G., et al., 1997, A\&AS (in press)

Puy D.L. du, 1970, AJ 75, 1143

Schneider S.E., Helou G., Salpeter E.E., Terzian Y., 1986, AJ 92,742

Schneider S.E., Thuan T.X., Mangum J.G., Miller J., 1992, ApJS 81, 5

Shuder J.M., Osterbrock D.E., 1981, ApJ 250, 55

Stoke J.T., Tift W.G., Kaftam-Kassim M.A., 1978, AJ 83, 322

Strauss M.A., Huchra J.P., 1988, AJ 95, 1602

Strauss M.A., Huchra J.P., Davis M., Yahil A., Fisher K.B., Tonry J.L., 1992, ApJS 83, 29
Tifft W.G., 1982, ApJS 50, 319

Takase, B., Miyauchi-Isobe N., 1984, Ann. Tokyo Astron. Obs. 2nd Ser. 19, 595

Tamazian V.S., 1983, Soobshch. Byurakan Obs. 56, 80

Tamazian V.S., 1984, Astrofizika 20, 43

Tamazian V.S., 1987, Astrofizika 26, 411

Theureau G., Bottinelli L., Coudreau N., et al., 1997, A\&AS (in preparation)

Vaucouleurs G. de, et al., 1991, Third Reference Catalogue. Springer-Verlag

Vaucouleurs G. de, Vaucouleurs A. de, Nieto J.-L., 1979, AJ 84,1811

Weedman D.W., Khachikian E.Y., 1969, Astrofizika 5, 113

Wegner G., Haynes M.P., Giovanelli R., 1993, AJ 105, 1251 
$M-2-2-38$

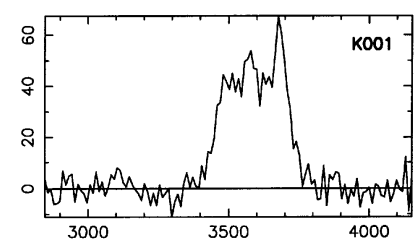

KAZ 6

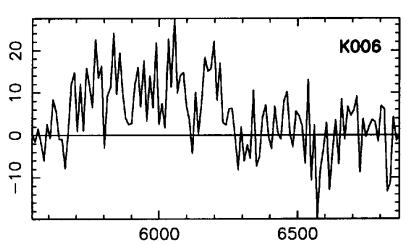

N 29
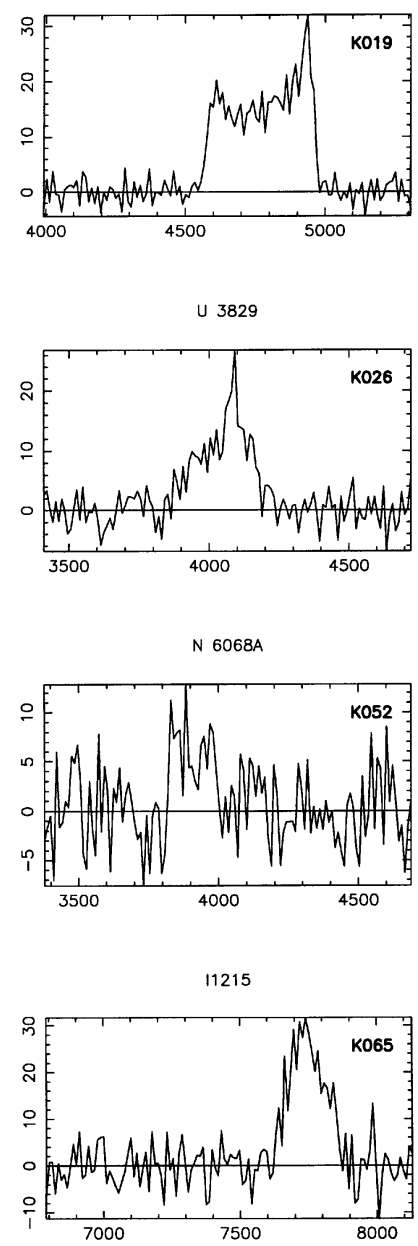

I 56

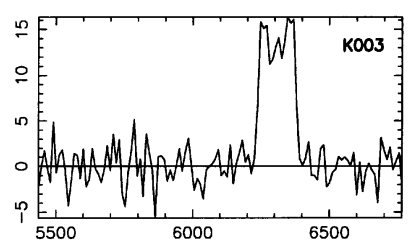

M 4-1-20

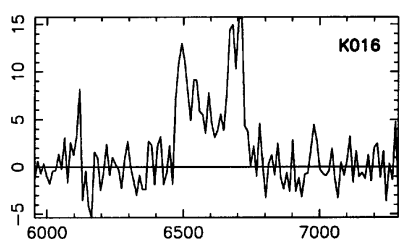

U 238

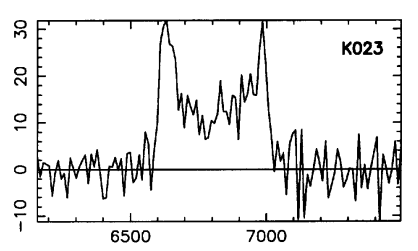

$M 12-15-20$

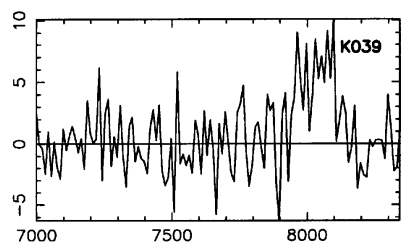

ZW 320-9
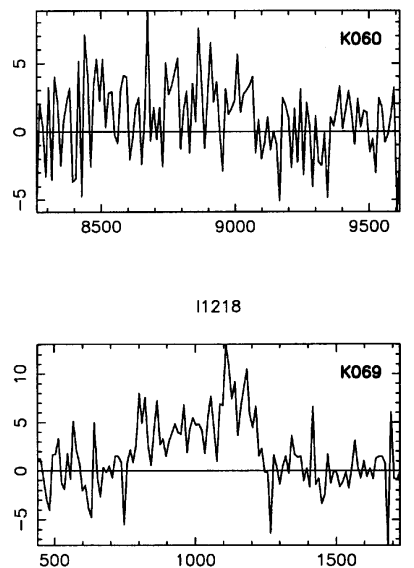

I $56 \mathrm{~A}$

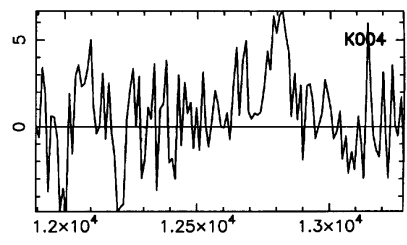

N 21

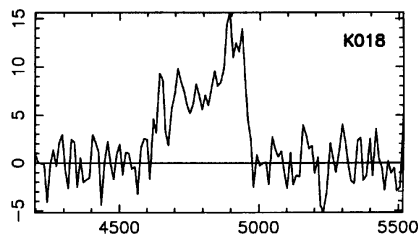

N 112

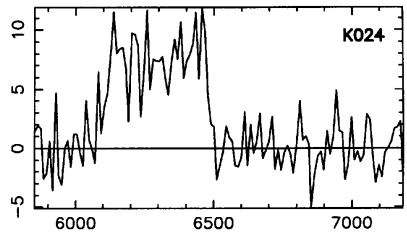

KAZ 47

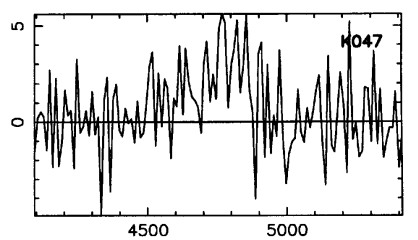

KAZ 63
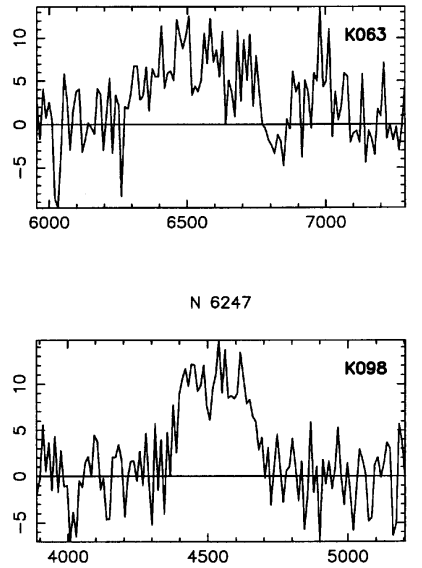

Fig. 2. HI spectra for 39 Kazarian galaxies: HI flux density in mJy is plotted against radial velocity in $\mathrm{km} \mathrm{s}^{-1}$ expressed in terms of optical redshift $\frac{c \Delta \lambda}{\lambda_{0}}$ 

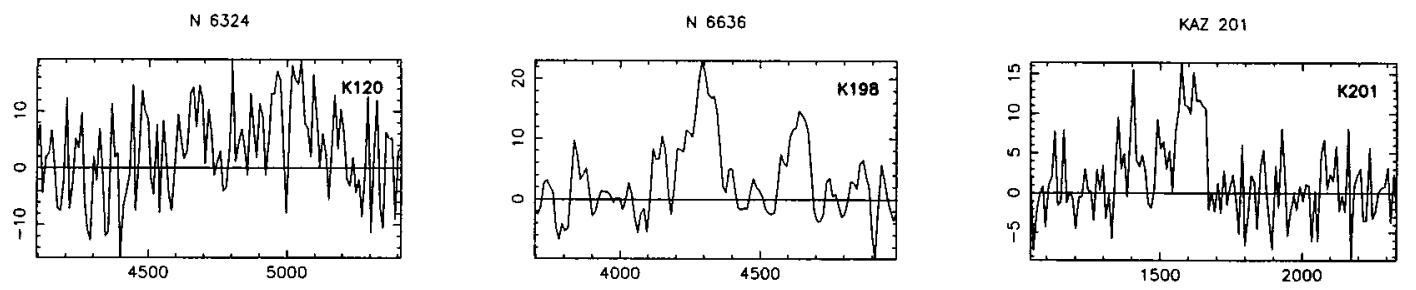

ZW $340-51$

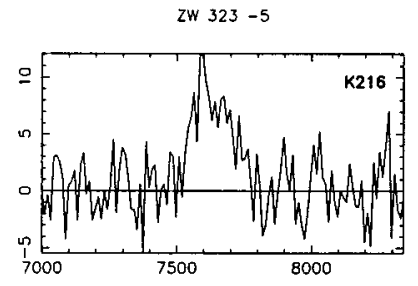

N 7324
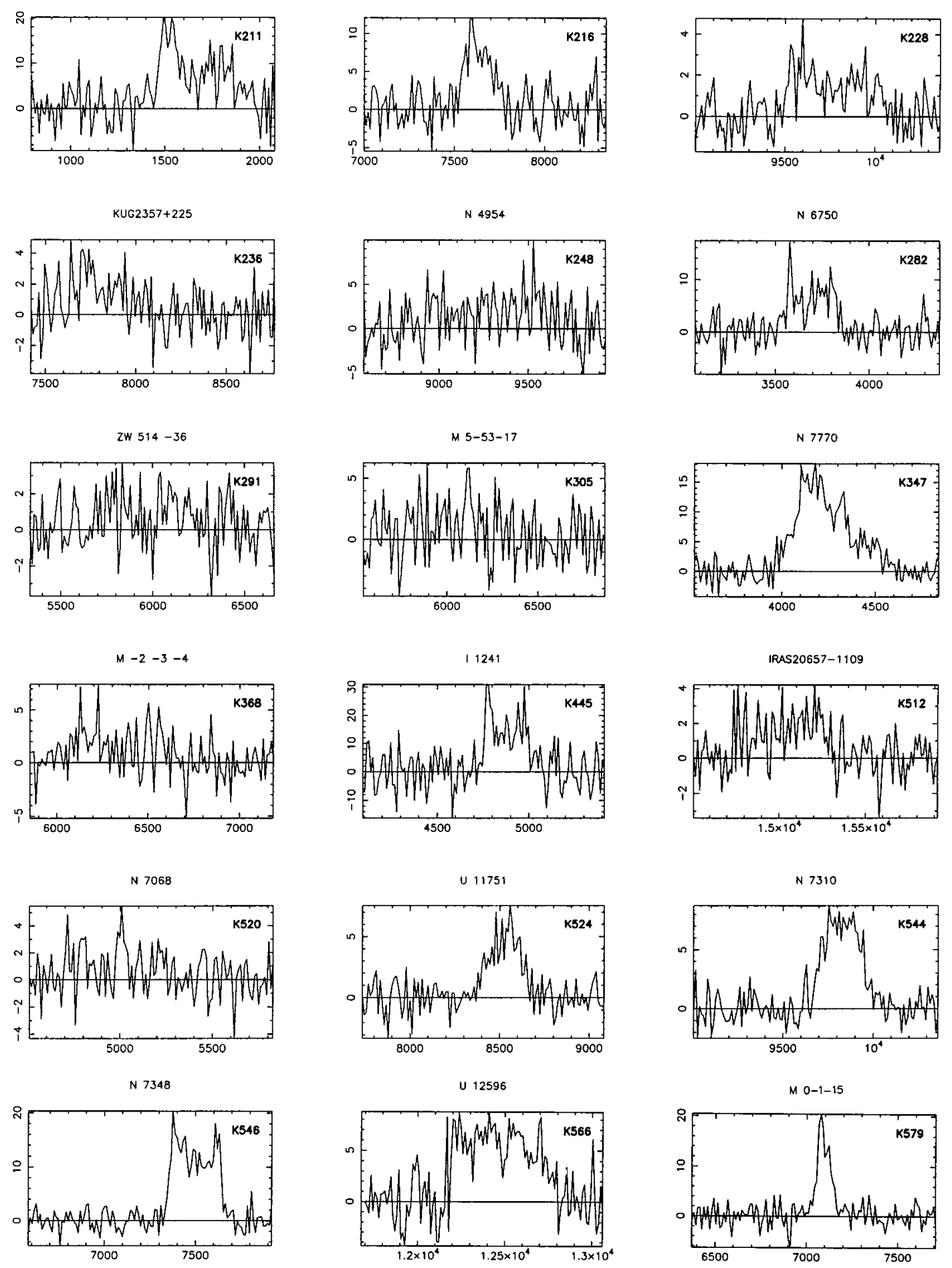

Fig. 2. continued 